\title{
Computer Simulations of "Realistic" Microstructures Using Statistical Two- Point Correlation Functions
}

\author{
A. M. Gokhale, H. Singh \\ School of Materials Science and Engineering, Georgia Institute of Technology, Atlanta, GA-30332
}

Two-point, three-point, and higher order microstructural correlation functions are important class of statistical descriptors that are useful for characterization of spatial arrangement and heterogeneity of microstructural features [1]. These correlation functions also contain quantitative information concerning the metric microstructural properties such as volume fractions of the phases, topological properties such as number and connectivity, as well as morphological anisotropy. Therefore, they are suitable for representation of microstructural geometry and useful for computer simulations of realistic microstructures that are statistically similar to the corresponding real microstructures. In a three-dimensional (3D) microstructure containing two phases, say particles (phase-1) and matrix (phase-2), the two-point correlation function $\mathrm{P}_{11}(\mathrm{r}, \theta, \phi)$ is the probability that both the end-points of a randomly located straight line of length $r$ and angular orientation $(\theta, \phi)$ are contained in phase-1 (i.e., particles). One can similarly define, $\mathrm{P}_{22}(\mathrm{r}, \theta, \phi)$, where both the end-points are in phase-2 (matrix), and $\mathrm{P}_{12}(\mathrm{r}, \theta, \phi)$, where one end of the line is in phase-1 and the other is in phase- 2 . Figure 1 illustrates these definitions. Although for a two-phase microstructure, there are four possible two-point correlation functions, only one of the four is independent. In an earlier contribution [2], an unbiased, efficient, and robust practical technique has been presented for estimation of direction dependent as well as orientation averaged two-point correlation functions in three-dimensional microstructures from the measurement performed on vertical metallographic planes. It has been shown that numerous length scale parameters that characterize spatial heterogeneity and clustering can be extracted from the two-point correlation functions [2].

In this contribution, we present a new methodology for computer simulations of "realistic" microstructures, where the features in simulated microstructures have the same complex morphologies as those in the corresponding real microstructures. Further, the two-point correlation functions, feature size and shape distributions, morphological anisotropy, and metric microstructural properties of the simulated microstructures are in close agreement with the corresponding properties of the real microstructures. The simulation algorithm is similar to Monte Carlo simulations involving iterative steps. These simulations capture all relevant details of the microstructural geometry in terms of few simulation parameters. The variations of these parameters can be then used to simulate the variations in the processing conditions of the material. The simulation parameters can be also used for microstructure-properties relationships and simulated microstructures can serve as representative macrostructure volume element (RVE) for the finite elements (FE) based and other computational techniques for simulations of materials behavior. An example of the new microstructure simulation methodology is depicted in Figure 2, where the real microstructure of a metal matrix composite (MMC) containing SiC particles (not fibers) of irregular shapes in an Al-alloy matrix (Figure 2a and 2c) is simulated (Figure 2b and 2d) via matching their two-point correlation functions [2]. Note that the $\mathrm{SiC}$ particles in the simulated microstructure have the same irregular complex morphologies and size distribution as those in the corresponding real microstructure, and the simulation mimics the spatial clustering of the $\mathrm{SiC}$ particles as observed in the real microstructure. 


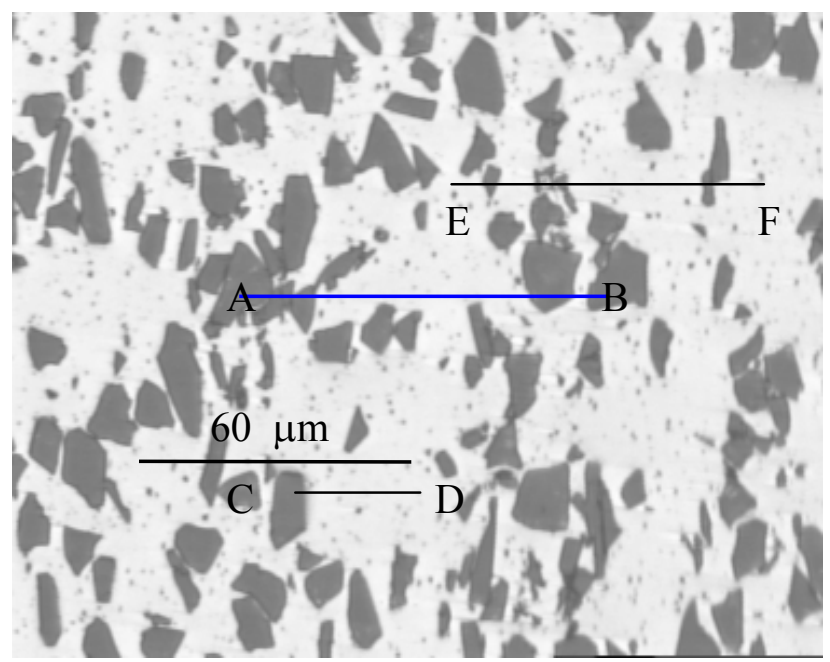

FIG 1: Two-point correlation functions: line $\mathrm{AB}: \mathrm{P}_{11}(\mathrm{r}, \theta, \phi)$, line $\mathrm{CD}: \mathrm{P}_{12}(\mathrm{r}, \theta, \phi)$, and line EF: $\mathrm{P}_{22}(\mathrm{r}, \theta, \phi)$

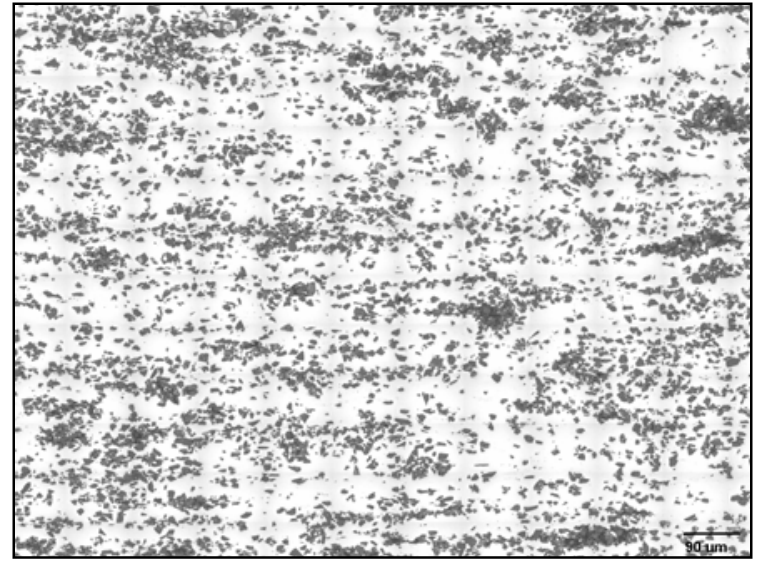

FIG 2a: Real microstructure of MMC

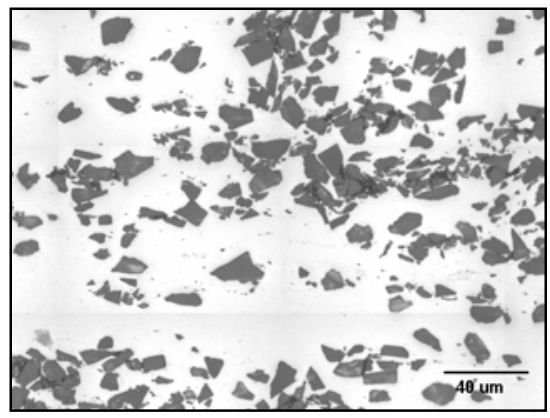

FIG 2c: High magnification view of $2 \mathrm{a}$.

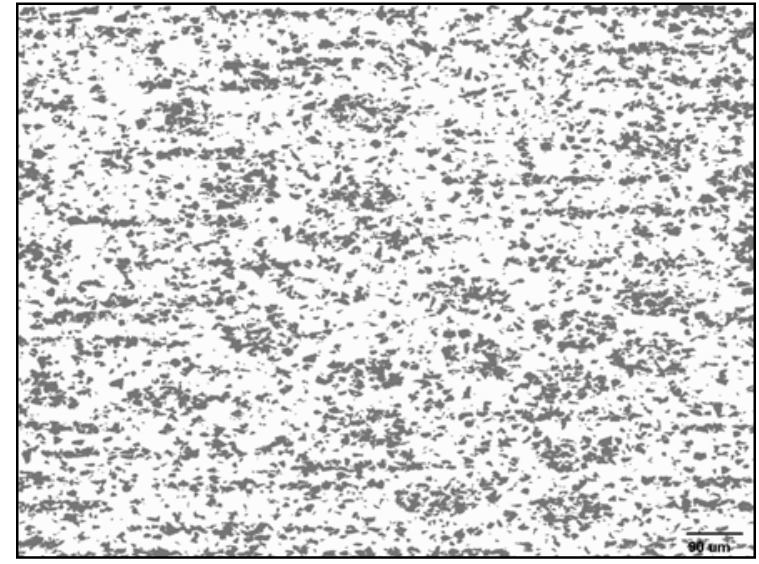

FIG 2b: Simulated microstructure of MMC

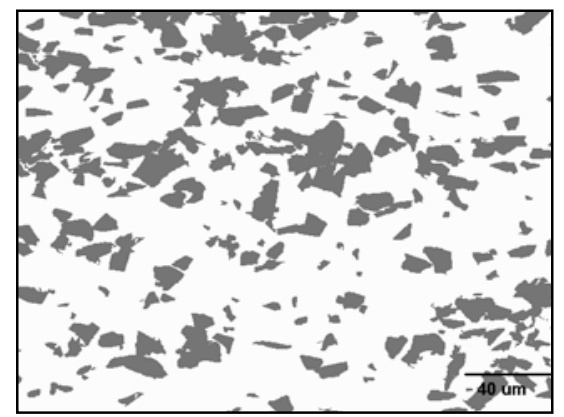

FIG 2d: High magnification view of $2 \mathrm{~b}$

\section{References}

[1] D. Stoyan, S. Kendall, and J. Mecke, Stochastic Geometry and its Applications, Second Edition, John Wiley and Sons, NY, 1995.

[2] A. Tewari, A.M. Gokhale, J. E. Spowart, and D. B. Miracle, Acta Materialia, 52 (2004)307.

[3] Acknowledgement: The U.S. National Science Foundation (grant DMR-0404668) and the Air Force Office of Scientific Research (grants F49620-01-1-0045 and 04-NA-152) supported this work. The financial support is gratefully acknowledged. 